\title{
Reply to the Letter to the Editors: DBS patient with diagnosed Non-Hodgkin's lymphoma: is radiation therapy safe?
}

\author{
Michał J. Schinwelski ${ }^{1}$, Jarosław Dulski ${ }^{2,3}$, Wojciech Łuczak $^{4}$, Witold Libionka, ${ }^{5,6}$, Tomasz Mandat ${ }^{7}$, \\ Jarosław Sławek ${ }^{2,3}$ \\ ${ }^{1}$ Neurocentrum-MiwoMed - Neurological Clinic, Gdansk, Poland \\ ${ }^{2}$ Neurology Department, St. Adalbert Hospital, Gdansk, Poland \\ ${ }^{3}$ Neurological and Psychiatric Nursing Department, Medical University of Gdansk, Gdansk, Poland \\ ${ }^{4}$ Oncology and Radiotherapy Department, Oncology Centre of Gdynia, Gdynia, Poland \\ ${ }^{5}$ Neurosurgery Department, M. Kopernik Hospital, Gdansk, Poland \\ ${ }^{6}$ Department of Neurobiology of Muscle, Faculty of Rehabilitation and Kinesiology, Gdansk University of Physical Education and \\ Sport, Gdansk, Poland \\ ${ }^{7}$ Department of Neurosurgery, Maria Sklodowska-Curie Memorial Oncology Centre, Warsaw, Poland
}

(Neurol Neurochir Pol 2020; 54 (4): 358-359)

We thank Drs. Milanowski, Grassle and Uitti for their interest in our article [1]. We agree that it is important to report every new patient who requires radiation therapy (RT) with implanted deep brain stimulation (DBS). As the number of DBS patients with concomitant cancer continues to rise, the influence of RT on those patients should be analysed.

At the time of qualification for DBS, both of our patients had cancer histories: the first patient had been diagnosed with prostate cancer and was undergoing hormone therapy, stable on urological examination and biochemical markers; the second patient had a history of left-sided mastectomy due to breast cancer. Such patients may be considered as being at risk if they were to encounter future RT. Important factors determining the risk of harmful interference from RT to DBS include the incidence of specific cancer type and its localisation. The reported DBS patient with non-Hodgkin's lymphoma affecting the lymph node in the vicinity of the internal pulse generator (IPG) represents an important clinical dilemma [2]. This neoplasm is listed by the American Cancer Society as being one of the most common cancers in the United States, and RT is a viable treatment option [3]. Other common cancers that may interfere with the DBS system, especially with pulse generators that are usually placed in the infraclavicular region, include lung and breast cancer. The latter was the culprit in our second patient, in whom RT of the left subclavian area with $15 \mathrm{MV}$ photons and a dose of
$20 \mathrm{~Gy}$ in five fractions was carried out (Fig. 1). The maximal estimated radiation dose for left IPG was 1.7 Gy. In a case report by Brokenhagen et al., the maximal radiation dose for IPG was over $48 \mathrm{~Gy}$.

Broader experience with pacemakers and implantable cardioverter defibrillators (PM/ICD) has shown that the impact of RT on a device depends on the beam energy rather than on the total dose of radiation [4]. In the two aforementioned cases, the maximal recommended beam energy $(<10 \mathrm{MV})$ and maximal recommended radiation dose were far exceeded, but fortunately no complications from IPG were observed. The surgical relocation of IPG is an option for minimising its exposure for RT [5], but possible malignant DBS withdrawal syndrome associated with acute cessation or failure of the DBS device must be considered [6]. In addition, maintaining neurostimulation during an RT procedure is crucial to avoid unwanted involuntary movements such as the head tremor in the case reported by Mazdai et al. [7].

In summary, despite the lack of structured studies, RT has been safely delivered in all patients with DBS reported thus far. Nonetheless, further multidisciplinary efforts should be made so as to ascertain the safety and feasibility of radiotherapy in patients with implanted DBS.

Conflict of interests: None.

Funding: None. 


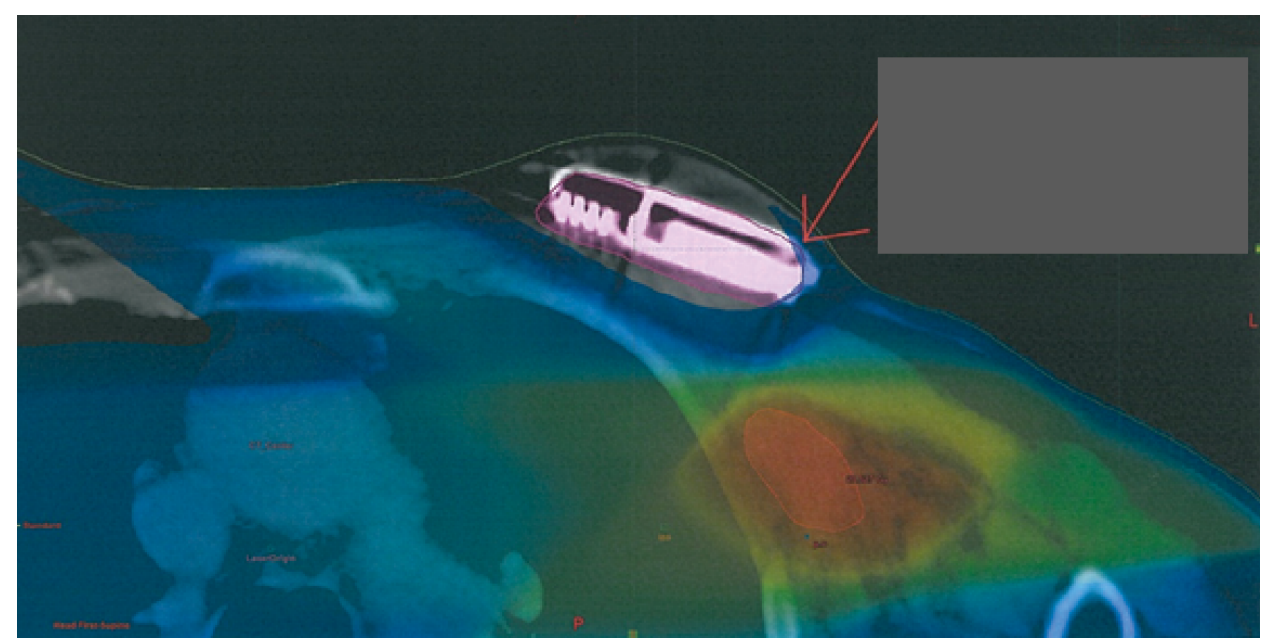

Figure 1. Planning of radiotherapy of breast cancer near pulse generator in Patient 2. Maximal estimated radiation dose for left internal pulse generator (IPG) was 1.7 Gy [1]

\section{References}

1. Schinwelski MJ, Dulski J, Łuczak W, et al. Radiation therapy in patients with implanted deep brain stimulation. Neurol Neurochir Pol. 2020; 54(3): 280-283, doi:10.5603/PJNNS.a2020.0033, indexed in Pubmed: 32285432.

2. Milanowski L, Grassle AL, Uitti RJ, et al. DBS Patient With Diagnosed NonHodgkin Lymphoma: Is Radiation Therapy Safe? Neurol Neurochir Pol. 2020; 54(4): 356-357, doi: 10.5603/PJNNS.a2020.0051, indexed in Pubmed: 32677029.

3. Key Statistics for Non-Hodgkin Lymphoma. www.cancer.org (24 August 2019).

4. Zaremba T, Jakobsen AR, Søgaard M, et al. Radiotherapy in patients with pacemakers and implantable cardioverter defibrillators: a literature review. Europace. 2016; 18(4): 479-491, doi: 10.1093/ europa-ce/euv135, indexed in Pubmed: 26041870.
5. Son BC, Kim JS, Park WC, et al. Management of Pulse Generators in a Breast Cancer Patient with in Situ Subthalamic Nucleus Deep Brain Stimulation. J Neurol Surg A Cent Eur Neurosurg. 2019; 80(3): 223-227, doi: 10.1055/s-0038-1677518, indexed in Pubmed: 30708388.

6. Neuneier J, Barbe MT, Dohmen C, et al. Malignant deep brain stimulation-withdrawal syndrome in a patient with Parkinson's disease. Mov Disord. 2013; 28(12): 1640-1641, doi: 10.1002/mds.25494, indexed in Pubmed: 23857820.

7. Mazdai G, Stewart DP, Hounsell AR. Radical radiation therapy in a patient with head and neck cancer and severe Parkinson's disease. Clin Oncol (R Coll Radiol). 2006; 18(1): 82-84, doi: 10.1016/j. clon.2005.09.009, indexed in Pubmed: 16477925. 